\title{
Coming to 'Terms' with the Journal
}

\author{
Wendell Roelofs
}

Published online: 17 May 2014

(C) Springer Science+Business Media New York 2014

The 40th anniversary of the Journal of Chemical Ecology has provided an opportunity to rise above the bliss of retirement and think back to what was happening in chemical ecology in the distant past. It was certainly an exciting time at Cornell with the recognized world leaders in the field, Drs. Jerry Meinwald and Tom Eisner, as colleagues. Research on pheromones was expanding exponentially, particularly as insect chemical communication systems were rapidly being decoded. A brief scan of my publications in the years prior to 1975 exposed a problem that developed as research efforts in this area sprung up around the world. It was relatively easy to publish a few new pheromone structures in Science and Nature in those early years, but many of my pheromone papers were published in a hodgepodge of journals, including Environ. Entomol., Ann. Entomol. Soc. Amer., Bull. Soc. Entomol., J. Econ. Entomol., Can. Entomol., Ent. Exp. Appl., Environ. Lett., Life Sci., J. Insect Physiol., and Experientia. It was not easy at that time to scan abstracts for 'pheromones' in all journals, so much time was consumed in looking at the Table of Contents of many journals for the publication of new pheromone papers. This included journals published in foreign languages that might contain an important article. Founding of the new Journal of Chemical Ecology was a tremendous solution to this problem. This journal provided a place for scientists around the world to publish their findings in a journal that specialized in this exciting scientific field. I loved the journal so much that in its first year our group published 4 papers on new pheromone identifications and 1 paper on an in-depth study with Ring Carde and Tom Baker on the ethological function of the oriental fruit moth pheromone components.

The long-term success of this journal can certainly be attributed to the excellent leadership provided for many years by Milt Silverstein \& John Simeone, and then continued in great fashion by Jim Nation, David Jones and now by John Romeo. The tone was set in the early days that this was a journal that was open to a wide range of ideas and data in the field of chemical ecology. The journal has matured in many ways throughout the years and now has set a high standard that is reflected in a $70 \%$ rejection rate. Many papers published in the early years could be classified today as preliminary and having unsubstantiated data, but the whole field was undergoing growing pains. A majority of papers dealt with insect pheromones, which was a relatively new and undefined field. Manuscript reviewers had a difficult time since it seemed as though every scientist had different terms and different criteria for their conclusions. If 3 compounds were found to be active in a female moth gland, were they each referred to as a

W. Roelofs $(\square)$

Cornell University, Geneva 14456, NY, USA

e-mail: wlr1@cornell.edu pheromone? It was decided that a pheromone is the whole blend of active compounds and each chemical in the blend was referred to as a pheromone component. If 10 compounds were found to be present in an active extract from a female moth gland, were they all called pheromone components? It was decided that a chemical was only a pheromone component if it could be structurally characterized as a compound in the female sex pheromone gland and shown that it elicits a positive response from conspecific male moths by itself or when added to other pheromone components. What about compounds that decreased trap catch in field studies when added to a pheromone lure? They were called 'inhibitors', 'repellents', 'anti-attractants', etc. The terms were the subject of much discussion since scientists used different terms in their manuscripts for describing the same phenomenon of decreasing trap catch. Trap catch alone could not be used to describe behavioral or neurological effects on a responding male, so terms that implied a specific effect were not appropriate, which led to an agreement that 'antagonist' was a good term for those compounds.

A major struggle in nomenclature was the attempt to classify what could be called a pheromone and what was a sex attractant. The terms were loosely used in the early papers and seemed to be interchangeable. After many discussions at scientific meetings, it was concluded that a chemical could only be called a pheromone component if there were solid data on both the chemistry and biological activity of the compound. Field trapping studies were accepted for biological activity if the data were statistically significant. If a compound was found to be active in field trapping studies in capturing a significant number of males compared to check traps, but was not identified from the females, it was referred to as a sex attractant. The combination of pheromone identification studies and field-trapping test revealed that many species use the same compounds in different blends. This eventually put pressure on reviewers to determine what was exciting enough for a scientific paper in the Journal of Chemical Ecology. Finding a common pheromone compound in yet another species was not very exciting anymore. Finding a complex new structure was exciting.

Characterization of insect pheromones might have dominated the early days of the Journal of Chemical Ecology, but exciting contributions from the multifaceted discipline of chemical ecology brought the journal to new heights. It became obvious that we are still just scratching the surface in deciphering the interactions of organisms in nature. Although the chemical structures in animals and plants might be found in a small number of groups of mediating molecules, structural modifications to each biosynthetic theme have led to hundreds of thousands of individual compounds in nature. Research efforts from the molecular to whole organismal levels provide endless challenges at all levels and will provide the basis for a tremendous array of interesting papers for a long time in the Journal of Chemical Ecology. 